\title{
Microwave Dielectric Absorption Spectroscopy Aiming at Novel Dosimetry Using DNAs
}

\author{
Yoshinobu Izumi ${ }^{1 * *}$, Makoto Hirayama', Youichirou Matuo', Takeyoshi Sunagawa² \\ ${ }^{1}$ Research Institute of Nuclear Engineering, University of Fukui, Fukui, Japan; ${ }^{2}$ Fukui University of Technology, Fukui, Japan
}

\section{Original Research}

Received July 17, 2015

Revision August 21, 2016

Accepted November 28, 2016

Corresponding author:Yoshinobu Izumi

Research Institute of Nuclear Engineering, University of Fukui,1-2-4, Kanawa-cho, Tsuruga-city, Fukui 914-0055, Japan Tel: $+81-770-25-0021$

Fax: +81-770-25-0031

E-mail:y_izumi@u-fukui.ac.jp

This is an Open-Access article distributed under the terms of the Creative Commons Attribution NonCommercial License (http://creativecommons.org/ licenses/by-nc/4.0) which permits unrestricted noncommercial use, distribution, and reproduction in any medium, provided the original work is properly cited.

Copyright $\odot 2017$ The Korean Association for Radiation Protection

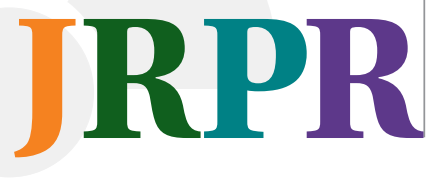

Background: We are developing L-band and S-band microwave dielectric absorption systems aiming novel dosimetry using DNAs, such as plasmid DNA and genomic DNA, and microwave technology.

Materials and Methods: Each system is composed of a cavity resonator, analog signal generator, circulator, power meter, and oscilloscope. Since the cavity resonator is sensitive to temperature change, we have made great efforts to prevent the fluctuation of temperature. We have developed software for controlling and measurement.

Results and Discussion: By using this system, we can measure the resonance frequency, $f$, and $\Delta \mathrm{Q}(\mathrm{Q}$ is a dimensionless parameter that describes how under-damped an oscillator or resonator is, and characterizes a resonator's bandwidth relative to its center frequency) within about 3 minutes with high accuracy.

Conclusion: This system will be expected to be applicable to DNAs evaluations and to novel dosimetric system.

Keywords: Microwave, Cavity, Dosimetry, DNAs, Radiation

\section{Introduction}

Nowadays, various kinds of dosimetry have been developed in order to evaluate the personal dose. Especially, dosimeters based on radiation physical process such as thermoluminescence dosimeter (TLD), optically stimulated luminescence (OSL) and radiation chemical process such as film badge are widely used $[1,2]$. They have good reproducibility and high accuracy with high sensitivity. On the other hand, many kinds of dosimeter have been developed and used in the field of radiation processing for industry, for example, ionization chamber (gas phase), Fricke dosimeter, cerium dosimeter (liquid phase), Cellulose Triacetate (CTA) film dosimeter, alanine dosimeter, semiconductor dosimeter (solid phase) [3].

Evaluation of personal dose is necessary to be able to measure the low-dose region with high sensitivity and high accuracy than dose assessment in radiation processing. On the other hand, we are considering that it is important to develop a new technology for personal dosimetry and to increase the measurement means.

We are aiming at developing novel dosimetry using deoxyribonucleic acids (DNAs) 


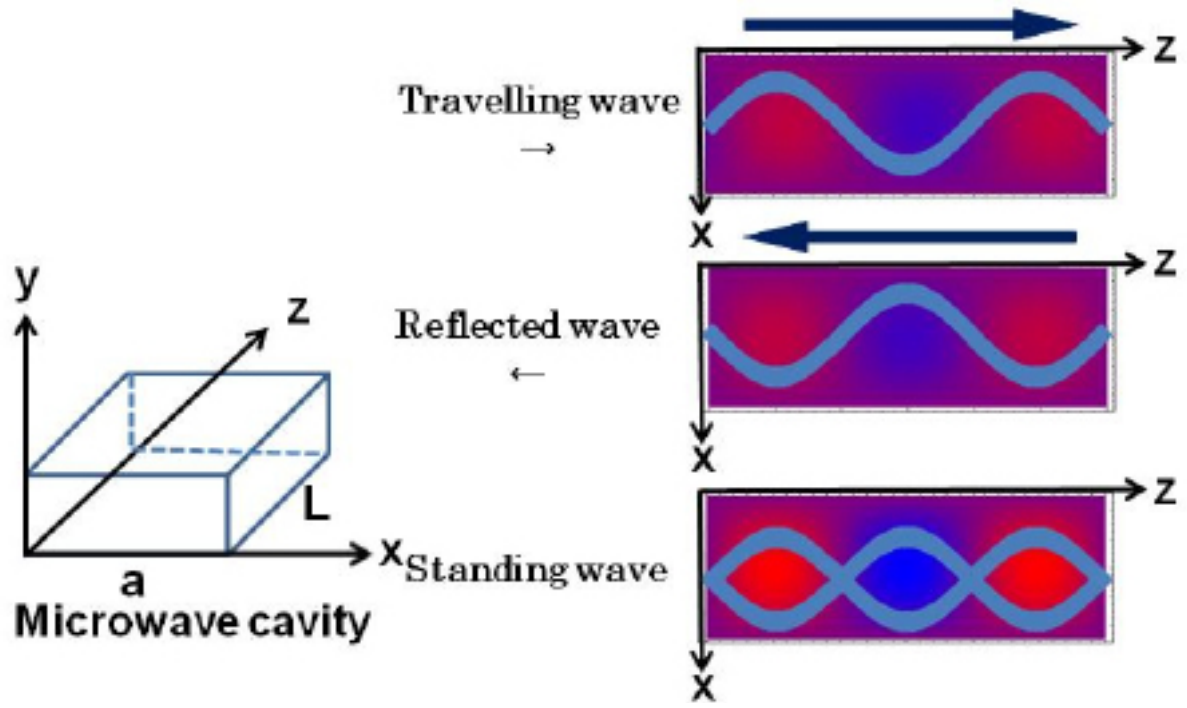

Fig. 1. Size of microwave cavity resonator and behavior of microwave in the cavity.

and microwave technology, and have installed L-band and S-band microwave cavity for the microwave dielectric absorption spectroscopy [4]. With inserting small amount of aqueous solution of DNAs in the position of electric field center of the cavity resonator, the resonant frequency of incident microwave is shifted and we can obtain the change in reflected power spectrum of the microwave. This methodology has advantage in sensitivity compared to conventional spectroscopic analysis based on the "Lambert-Beer's Law". Moreover, the spread of the line width of the spectrum in the reflected microwave, we can obtain information on the dielectric loss.

We have studied the fundamental characteristics of our system, such as influence of temperature, humidity etc. Also, we have studied the characteristic of response using various (polar and non-polar) liquids or mixed solution of water/ methanol with various concentrations as the samples.

In this paper, we describe the principle of this spectroscopic measurement, the structure and component of the system, and fundamental characteristics of developed system.

\section{Materials and Methods}

\section{Principle of microwave dielectric absorption spectroscopy}

When we prepare the rectangular micro wave cavity resonator with a size of $a \times b \times L$ as shown in Figure 1 (left) and inject the incident microwaves, only a combination of incident microwave and an reflected microwave with a frequency res- onant-able within the cavity can successfully form the standing wave as shown in Figure 1 (right). Resonance frequency of a rectangular microwave cavity for $\mathrm{TE}_{103}$ resonant mode can be found by imposing boundary conditions on electromagnetic field expressions. This frequency is given by,

$$
\boldsymbol{f}=\frac{c}{2} \sqrt{\frac{\left(\frac{3}{L}\right)^{2}+\left(\frac{1}{a}\right)^{2}}{\varepsilon}}
$$

where $f$ is frequency of resonant microwave, $\varepsilon$ is dielectric constant of materials filling in the cavity, and $\mathrm{c}$ is the speed of light in vacuum.

When certain amount of other material having other dielectric constant compared to filling material, air in this research, is inserted into the microwave cavity, in ideal case of at a center of electromagnetic field, the perturbation occurs and resonant frequency changes. If we measure the power spectrum of reflected microwave, we will be able to obtain power spectrum change.

The dielectric characteristics of the material can be expressed as,

$$
\varepsilon=\varepsilon^{\prime}-\mathrm{i} \varepsilon^{\prime \prime}
$$

where $\varepsilon^{\prime}$ is real part of dielectric constant, $\varepsilon^{\prime \prime}$ is imaginary part (dielectric loss) of dielectric constant. When certain kind of sample is inserted to cavity resonator, change in $\varepsilon^{\prime}$ and $\varepsilon^{\prime \prime}$ occurs and resulting dielectric constant $\mathcal{E}$ changes in the cavity resonator. Due to above-mentioned perturbation, output 

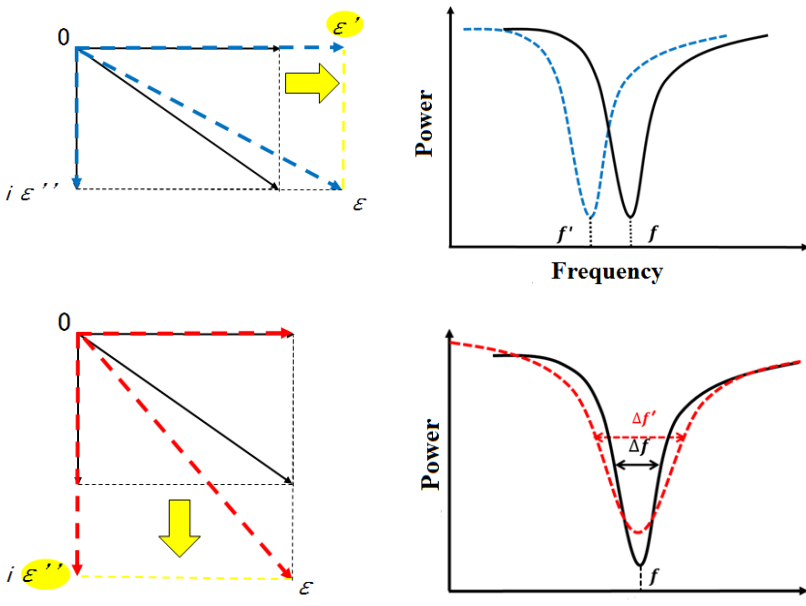

Frequency

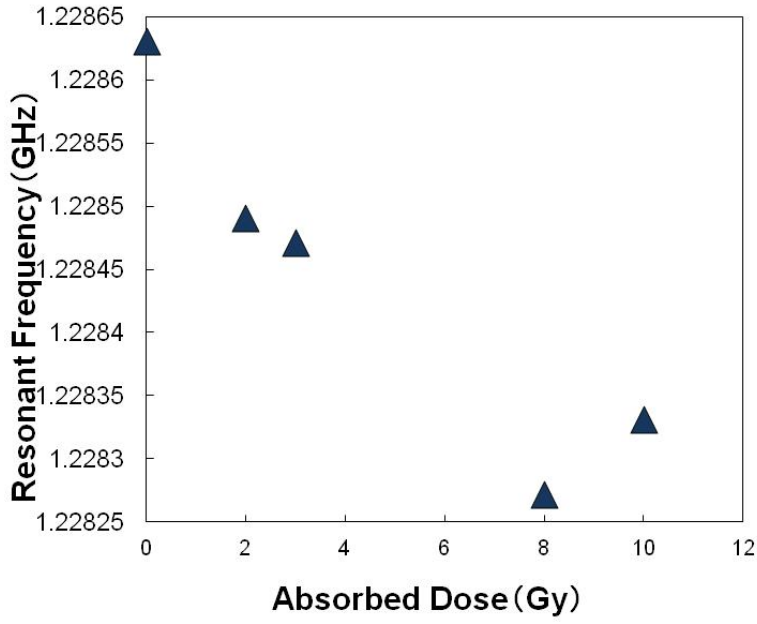

Fig. 2. The typical two cases of change in power spectrum. Upper: changing $\varepsilon^{\prime}$ corresponding to the change in the resonance frequency. Lower: changing $\varepsilon^{\prime \prime}$ corresponding to the inverse of $Q$-value.

signal of power spectrum changes by inserting certain amount of other material having other dielectric constant compared to filling air. Figure 2 shows typical two cases of change in power spectrum. One is the case of changing in the real part of the dielectric constant $\varepsilon^{\prime}$, corresponding to the change in the resonance frequency. The other is the case of changing in the imaginary $\varepsilon^{\prime \prime}$ corresponding to the inverse $\mathrm{Q}$-value. The Q-value is represented by,

$$
\text { Q-value }=f / \Delta f
$$

where $\Delta f$ is half power width. Therefore, we can know the changes in $\varepsilon^{\prime}$ and $\varepsilon^{\prime \prime}$ from the changes in resonance frequency and Q-value obtained from this microwave dielectric absorption measurement.

For example, when the plasmid DNA is selected as a sample, this DNA undergoes molecular chain scission, single- or double-strand break and resulting conformation change of molecule. Then $\varepsilon$ will change. Therefore, by measuring the changes in resonance frequency and Q-value by means of this microwave dielectric absorption spectroscopy, we can obtain the information about changes of $\varepsilon$ ' and $\varepsilon^{\prime \prime}$ and chain scissions (an example about the shift of resonant frequency by irradiation is shown in Figure 3).

\section{Measuring system of microwave dielectric absorption spectroscopy}

Figure 4 is the system of L-band microwave dielectric absorption spectroscopy. We have developed two systems,

Fig. 3. An example about the resonant frequency change with the gamma-irradiation to pUC118 plasmid DNA.

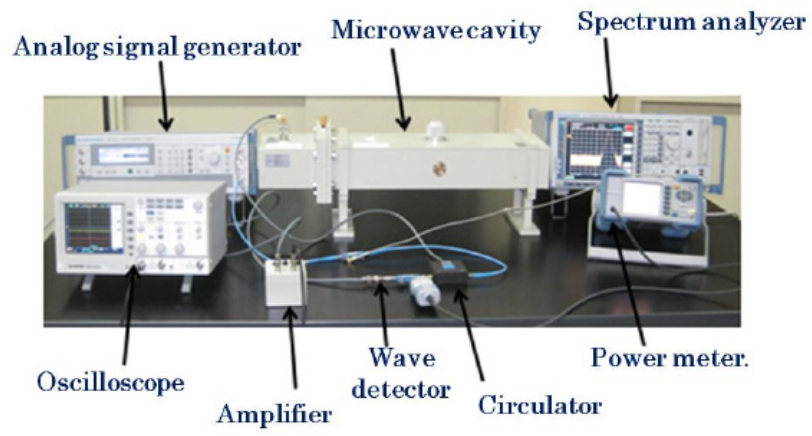

Fig. 4. The system of L-band microwave dielectric absorption spectroscopy.

namely L-band system with frequency region around 1.2 $\mathrm{GHz}$ and S-band system with frequency region around 2.3 $\mathrm{GHz}$ in order to provide better frequency from the viewpoints of sensitivity, accuracy and preventing dielectric loss due to water which is generally used as a solvent of bio-molecules. Each system is composed of a cavity resonator, analog signal generator, circulator, power meter, and oscilloscope. Microwave oscillated from an analog signal generator is led to the inside of the cavity resonator through a circulator. The power of the reflected wave is measured by the power meter while sending reflected wave from the cavity resonator inside to the oscilloscope, and observing a waveform of the reflected wave. In order to measure a resonance frequency and power of the reflected wave automatically and rapidly, all of these components were connected to and controlled by a personal computer. We have newly developed software for controlling and measuring. The duration to obtain the power spectrum, $f$ and $\Delta \mathrm{Q}$ is about 3 minutes. 


\section{Results and Discussion}

\section{Fundamental characteristics of developed system and further improvements}

In order to detect and analyze the slight amount of change of dilute DNA aqueous solution, for example the sample of dilute aqueous solution containing plasmid DNA (ex. See Figure 5) induced by irradiation, we must achieve a stable

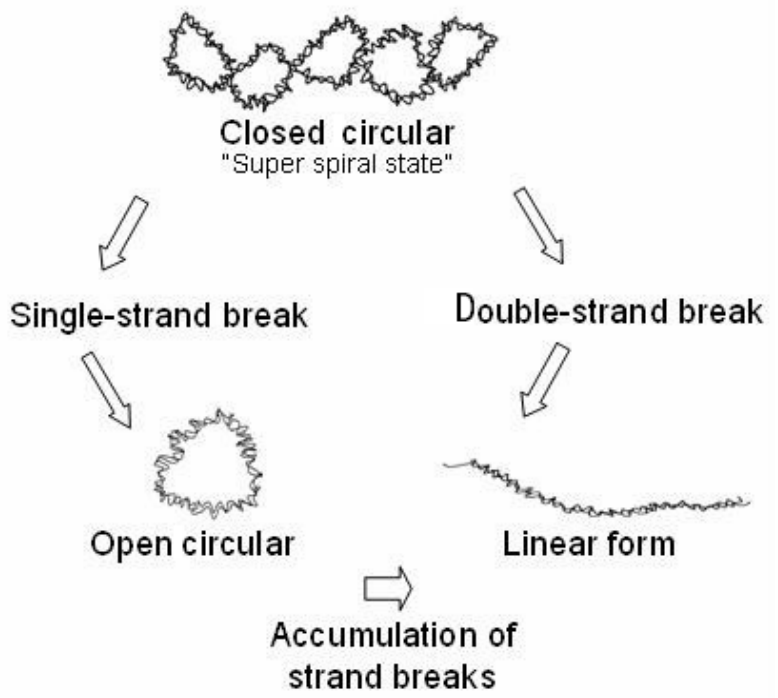

Fig. 5. Change in molecular structure of plasmid DNA caused by strand breaks. system having low effect of surrounding temperature, humidity etc., since the output signal of $f$ is dependent on the size of cavity and $\varepsilon$ as indicated by Equation 1 . The length of wall material of cavity may change depending on the temperature, and the average of $\varepsilon$ may change depending on the content of humidity. Therefore, first, we checked the relation between room temperature and $f$, and obtained the result; $f$ decreased with an increase of room temperature, the slope was estimated as $-7\left[\mathrm{kHz} \cdot \mathrm{K}^{-1}\right]$. Second, we confirmed the effect of humidity on $f$ was negligible. From these result, we improve the system adding the thermal insulation box to keep the temperature of cavity and purged with dry air to keep the humidity in the cavity. After this improvement we could establish more stable system for the application to analysis of DNA.

Concerning the estimation of $\Delta \mathrm{Q}$, the accuracy of measured $f_{1}$ and $f_{2}$ in Figure 6 is key-point since $\Delta \mathrm{f}$ is obtained from $f_{2}-f_{1}$. Therefore, we employed algorithm of a "hunting hound dog-like" method as shown in Figure 6 (left lower) and newly developed and improved the software for measurement. This "hunting hound dog-like" method is characterized in that the frequency of incident microwave is driven always from one direction, and when the frequency exceeds a target position, $f_{1}$ or $f_{2}$, the frequency is largely returned and then the frequency is driven again with a value smaller than

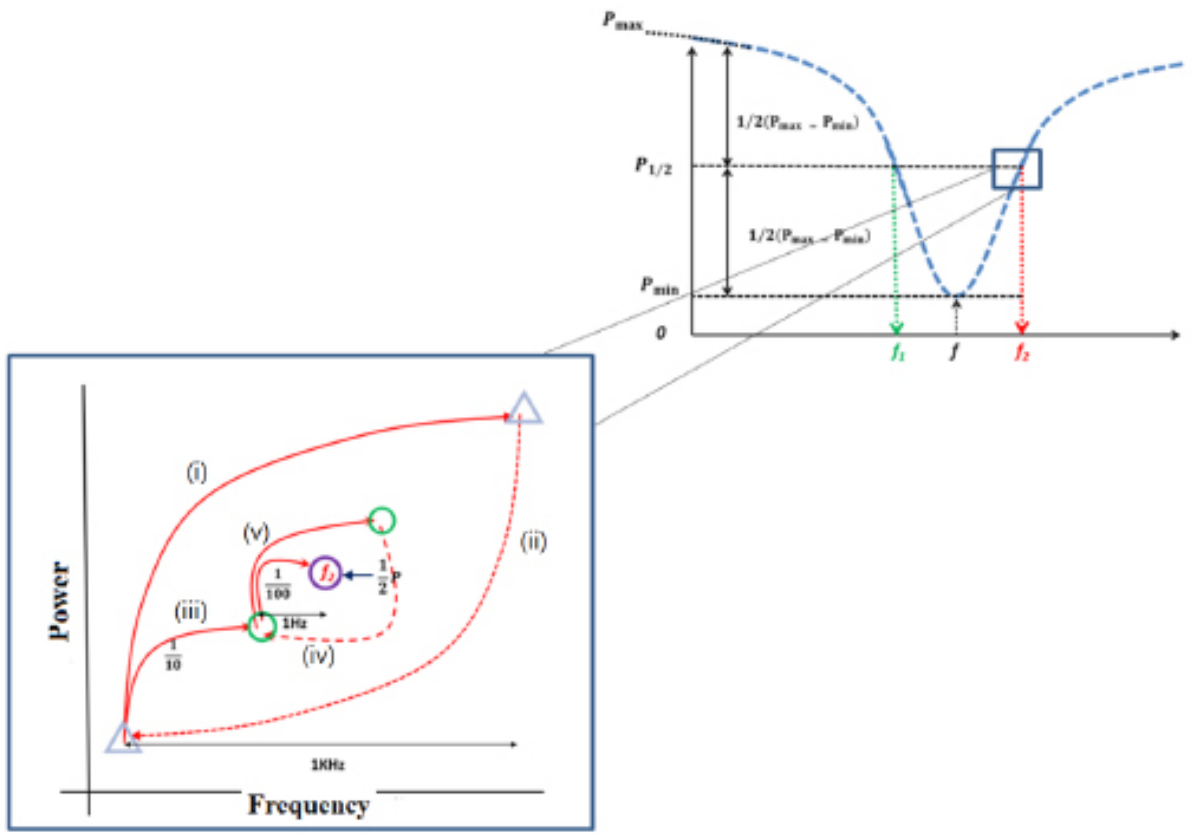

Fig. 6. The strategy and concept to obtain $\Delta$ Q. (i) The frequency of incident microwave is driven always from one direction. (ii) When the frequency exceeds a target position, $f 1$ or f2, the frequency is largely returned. (iii) And then the frequency is driven again with a value smaller than the preceding driving (stepping) value. (iv, v) Above-described procedures are repeatedly done with a smaller stepping value. 
the preceding driving (stepping) value.

After improvement and employment of this "hunting hound dog-like" method, we measure the response of L-band cavity with inserting $1.0 \mathrm{~mL}$ of distilled water as a sample 50 times to check the accuracy and reproducibility. Table 1 indicates the deviations of $f$ and $\Delta \mathrm{Q}$ obtained from 50 times of measurement of $1.0 \mathrm{~mL}$ water. Relative deviation of $\Delta \mathrm{Q}$ was successfully reduced to about $55 \%$ without any deterioration of accuracy of $f$ by employing "hunting hound dog-like" method. Therefore the reliability of this system was increased. The results of experiments with DNAs were reported in elsewhere [5]. We have made great effort to reduce the influences by conditions such as temperature and humidity. To achieve more accurate measurement of this method, we are planning to establish simultaneous measurement of temperature of microwave cavity and develop a new program for temperature compensation.

\section{Conclusion}

We have developed L-band and S-band systems of microwave dielectric absorption spectroscopy for the application to the evaluation of DNAs, and employed algorithm of a "hunting hound dog-like" method to achieve high accuracy and reproducibility without any deterioration of accuracy of resonant frequency. After more improvement of accuracy, sensitivity and stability, these systems can be expected to be applicable to dosimetric system with DNAs in the future.

\section{Acknowledgements}

This work was supported by JSPS KAKENHI Grant Numbers 24561039, 15K06666. This work was also supported by Fukui Prefecture and Fukui Prefectural Government's Grantin-Aid for Collaboration Research Projects.

\section{References}

1. Ledoux M. Nuclear engineering handbook, Chap. 25. 2nd Ed. Boca Raton, FL. CRC Press. 2015;913-916.

2. Tsoulfanidis N, Landsberger S. Measurement \& detection of radiation, Chap. 16.9. 4th Ed. Boca Raton, FL. CRC Press. 2015;503512.

3. Woods PJ, Pikaev AK. Applied radiation chemistry: Radiation processing, Chap. 4. 1st Ed. New York, NY. John Wiley \& Sons. 1994;90-125.

4. Grigas J. Microwave dielectric spectroscopy of ferroelectrics and related materials, Chap. 3. 1st Ed. Amsterdam, Netherland. Gordon and Breach Publishers. 1996;23-46.

5. Hirayama M., Matuo Y., Sunagawa T., Izumi Y., Evaluation of DNA damage using microwave dielectric absorption spectroscopy, J. Radiat. Prot. Res. 2016;41(4):339-343. 